\title{
Reducing Stigma Barriers to Help-Seeking Behaviors among College Students
}

\author{
Emily Reichert \\ Pennsylvania State University, University Park, USA \\ Email: ecr5068@psu.edu
}

Received July $18^{\text {th }}, 2012$; revised August $19^{\text {th }}, 2012$; accepted September $21^{\text {st }}, 2012$

\begin{abstract}
College students suffer disproportionately from depression, an illness with significant consequences that, untreated, escalates in severity. A review of literature reveals that seeking help for this health issue is often stigmatized, reducing the likelihood of treatment. While the literature identifies the types of stigma, less is known about the communicative processes involved in stigma coping. This paper applies Meisenbach's (2010) Theory of Stigma Management Communication (SMC) to this issue, suggested strategies researching depression stigma coping as well as new and promising intervention strategies to increase help seeking rates among college students.
\end{abstract}

Keywords: Depression; Stigma Management Communication; College Students; Help Seeking

\section{Introduction}

Depression, the most common psychiatric disorder and among the top causes of mortality worldwide, begins prior to age 24 in three fourths of all lifetime cases (Gladstone et al., 2011; Richards, 2011; Eisenberg et al., 2007). These observations are especially relevant to students attending college, as over one third of the United States college student population report depressive symptoms that, at the very least, interferes with their relationships, well-being, and school work (Zivin et al., 2009; Michael et al., 2006; NIMH; CDC, 2011; Eisenberg et al., 2007). Suicidal risk, the third leading cause of death for 15- to 24-year olds, is increased by depression and the rates of suicidal thoughts on college campuses range between $2.5 \%$ and 9\% (CDC, 2011; Eisenberg et al., 2007; Klein et al., 2011). While depression is among the most treatable mental illnesses, college students are the demographic most in need of help, but are the least likely to seek it, with only $3 \%-12 \%$ doing so (Gladstone et al., 2011; Ting, 2011; Richards, 2011; Zivin et al., 2009; Eisenberg et al., 2007; Michael et al., 2006; Rickwood, 2007).Instead most ignore the symptoms and rely on harmful self-medicating behaviors such as alcohol and drug use, all of which only increase symptom severity (Gladstone et al., 2011; Ting, 2011; Cassano \& Fava, 2002; Michael et al., 2006; Zivin et al., 2009; Rickwood, 2007; Klein et al., 2011). One might ask: why would one ignore symptoms with such severe potential consequences when proven treatment options are available through college campuses without any financial or accessibility obstacles? Most people are able to correctly identify symptoms of depression; therefore this disproportion in help seeking rates cannot be explained by lack of information (Elwy et al., 2011). The answer, it appears, is at least in part due to the stigma associated with depression status. Stigmastrongly correlates with decreased willingness to seek help and disclose information in the general population regardless of depression status, but particularly so for the college demographic(Schomerus et al., 2009b; Ting, 2011; Barney et al., 2011).Exploring stigma communication and possible responses to stigma is beneficial for affected individuals, health professionals, and the college population because it allows for recognition of stigma as a legitimate resource or hindrance to seeking help for depression, and how these issues can be addressed to improve help seeking rates (Boardman et al., 2011). Thus, it is imperative to consider stigma reduction programs and research and this involves a focus on how stigma is communicated. A literature review was conducted to elucidate the processes involved in help seeking, depression, and stigma among college students. Research published within the past decade was given priority and recommendations are then offered. The discussion starts with the concept of stigma.

\section{Stigma Research}

To say that depression and/or depression treatment are stigmatized means that there is a socially constructed, simplified, and standardized image used to mark those seeking treatment as "not normal" (Smith, 2007a; Goffman, 1963). Creation, perpetuation, and maintenance of this stigma depends on a communicative process involving cues (marks, responsibility, group labels, and peril) that serve to distinguish, categorize, blame, and link peril to the targeted group (Smith, 2007a). These cues dictate who is stigmatized, why they are stigmatized, and what constitutes stigmatization. They are commonly relied upon in communicative exchanges because of the accessibility and emotional arousing qualities that are useful for bonding and increasing popularity (Smith, 2007a).

The main normative factors influencing communication about depression stigma are public stigma, self-stigma and social distance. Public, or perceived, stigma is an individual's perception of what the general population believes about a stigmatized topic (Yap et al., 2011).

Self-stigma, the second factor, is an individual's application of public stigma to the self (Yap et al., 2011). One example of depression self-stigma is the perception that a mental illness signifies a personal weakness rather than an illness, and is found to decrease help seeking rates (Yap et al., 2011). In addi- 
tion to being associated with low help seeking rates, high selfstigmatization is also associated with decreased self-efficacy, decreased functioning, and increased hospitalization rates (Evans-Lacko, 2011). Finally, social distance is defined as the extent to which other individuals avoid contact with a stigmatized individual based on the stigmatized conception that depression is due to weakness of character (Yap et al., 2011; Jorm \& Griffiths, 2008).

There is a complex relationship between and among these factors. Public stigma does not directly influence help seeking decisions for depression but informs self-stigma and social distance that are both strongly related to help seeking intentions (Yap et al., 2011; Barney et al., 2006). Social distancing climates are associated with higher levels of self-stigma, suggesting that targeting social distance may be the most efficient way to combat depression stigma (Evans-Lacko et al., 2011; Schomerus et al., 2009b; Yap et al., 2011). The complexity of interactions between self-stigma and social distance along with contextual factors such as mental illness type, and demographic renders the task of studying coping with stigma a challenging one, but recent developments in stigma communication offer promising suggestions for where to begin and how to utilize current research to improve help-seeking rates.

\section{A Model of Stigma Communication}

Research has begun to identify strategies for coping with perceptions of stigma that can be applied to depression stigma (Meisenbach, 2010). Meisenbach's (2010) Theory of Stigma Management Communication (SMC) utilizes stigma attitudes from an individual as they apply to the self (the degree of selfstigma) and as the individual accepts or challenges the public's understanding of the stigma (relating to social distance). These two planes provide a framework for mapping stigma management in order to quantify and qualify efficacy in a systematic way that has not been possible before. However, the process is complex, with the model acknowledging that stigma statuses can vary across perceptions, discourse, time, and severity, and because of this fluidity the origin of the stigma under examination must be specified (Meisenbach, 2010).

SMC divides responses to stigma into four categories that re- flect the degree to which an individual internalizes the stigma (self acceptance) as well as the degree to which they agree with the public stigma messages (public acceptance). In other words they can either accept or challenge stigma internally as well as the public perception resulting in four over-arching categories or quadrants reflected in Figure 1 (e.g., accept internally/accept publicly; accept internally/challenge publicly; challenge internally/accept publicly; and challenge both internally and publicly). SMC then describes six main coping strategies for these four situations (see Figure 1 for more detail): accepting, avoiding, evading responsibility, reducing disgust, denying, and ignoring (Meisenbach, 2010).

\section{Quadrant 1: Accept Internally/Accept Publicly}

When stigma is accepted both internally and publicly the individual can accept the stigma through submissive means or in an active manner with humor, displaying, disclosing, apologizing, blaming, isolation, or bonding with other stigmatized individuals.

\section{Quadrant 2: Challenge Internally/Accept Publicly}

Here stigma is internally challenged but publicly accepted by distancing the self in various ways from anything attributable to the stigma whether through hiding, denial, avoidance, stopping behaviors, distancing, or making favorable comparisons.

\section{Quadrant 3: Accept Internally/Challenge Publicly}

Strategies falling in this quadrant accept the applicability of the stigma to the self but challenge the public's perception through strategies that shift agency away from the self or aim to change the public perception.

\section{Quadrant 4: Challenge Internally/Challenge Publicly}

When stigma is challenged in both dimensions the two available coping strategies include denial, which is divided into simple denial and logical denial (further divided into discrediting, providing evidence, or highlighting fallacies), and ignoring its expression.

\begin{tabular}{|c|c|c|c|}
\hline & & \multicolumn{2}{|c|}{ Stigma Application to the Self } \\
\hline & & Accept Internally & Challenge Internally \\
\hline \multirow{4}{*}{ 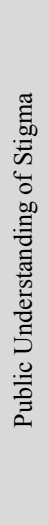 } & \multirow[b]{2}{*}{ Accept Publicly } & Quadrant 1 & Quadrant 2 \\
\hline & & $\begin{array}{ll}\text { - } & \text { Accept Stigma } \\
\circ & \text { Submissive } \\
\circ & \text { Active }\end{array}$ & - Avoid Stigma \\
\hline & & Quadrant 3 & Quadrant 4 \\
\hline & Challenge Publicly & $\begin{array}{l}\text { - Evade Responsibility } \\
\circ \text { Cause } \\
\text { - Provocation } \\
\circ \text { Control Intentionality } \\
\text { - Reduce Disgust } \\
\circ \text { Bolster alternative identity } \\
\circ \text { Minimize effects } \\
\circ \text { Transcend }\end{array}$ & $\begin{array}{ll}\text { - } & \text { Deny Stigma } \\
\circ \quad \text { Simple } \\
\circ \quad \text { Logical } \\
\text { - } & \text { Ignore Stigma }\end{array}$ \\
\hline
\end{tabular}

Figure 1.

Adapted from Meisenbach's (2010) model of stigma management communication (SMC). 


\section{Factors Impacting Strategy Choice}

Like stigma itself, decisions about coping are complex. However, research suggests that one of the keys to this process is the degree to which individuals accept personal responsibility for the stigma. People who accept personal responsibility have internalized public and social stigma cues that communicate blame towards stigmatized individuals. This, in turn, decreases help seeking intentions, and decreases empathy towards other group members (Smith, 2007a; Rickwood, 2007; Chang, 2008). Responsibility is broken into choice and control, one being the individual's responsibility in the onset of the stigmatized condition, the other being the amount of control an individual has over eliminating the condition (Smith, 2007a). Illnesses such as depression are stigmatized in both ways but are especially branded as more manageable than physical illness and will eventually go away if the individual takes control of the symptoms (Chang, 2008). The amount and type of responsibility that a stigmatized depressed person feels, therefore, impacts their coping strategy decision. For example, strong feelings of control responsibility correspond to an acceptance of the public understanding of depression stigma, therefore, depending on how the individual accepts or challenges that public understanding internally the coping strategy could result in either accepting or avoiding. The opposite would then be challenging the public perception, and depending on the acceptance/denial to the self, an individual could use evading responsibility or denial methods.

\section{Strategy Effectiveness}

Unfortunately, there is very little research regarding what coping methods are effective in increasing help seeking for depression, but what little there is has focused on the use of evading responsibility through defeasibility and provocation. Evading responsibility in SMC results from an acceptance of the stigma to the self but challenges the public understanding of stigma and is done through provocation, defeasibility, or unintentional means (Meisenbach, 2010). Defeasibility as applied to depression stigma relates directly to relinquishing control responsibility through admission that the individual could not avoid this stigma and that this strong need assume responsibility for their recovery simply is not a viable option (Meisenbach, 2010).Using defeasibility has not only been found effective, but also integral to increasing help-seeking intentions (Boardman et al., 2011). Having a strong sense of control responsibility is described as a cage that prevents help-seeking behaviors because holding onto the responsibility does not carry the risk of being personally scrutinized by the self and others as letting go does (Boardman et al., 2011).

Cause responsibility can be evaded from depression stigma through the use of provocation, or the reasoning that predetermined factors provoked the stigmatized state independent of the stigmatized individual's responsibility (Meisenbach, 2010). One way to employ provocation is through the use of scientific evidence such as biological information and, when applied to depression stigma, has been found to increase help-seeking intentions in college student populations (Han et al., 2006). Additionally, this strategy is thought to be effective for perceptions of depression because evidence is perceived as physical, more empirical, and less questionable (Han et al., 2006). Brain imaging scans are one useful tool for demonstrating the physi- cal nature of depression because they visually demonstrate reward-related areas of the brain such as the amygdala, the mesolimbic dopamine system, the prefrontal cortex, and the striatum differing in reward sensitivity in depressed compared to nondepressed individuals (NIMH, 2011; Forbes \& Dahl, 2012; Hankin, 2006). Biological information such as brain imaging scans take cause responsibility away from the stigmatized individual and instead focus it on predetermined factors, such as brain chemistry, to demonstrate that depression is often provoked by risk factors that are often out of the individuals control.

Ineffective strategies also have been documented. In SMC decreasing the degree of disgust attached to a stigma is a general strategy choice that results from an acceptance of the stigma applicability to the self, but challenges the public understanding of that stigma (Meisenbach, 2010). There are three ways to reduce disgust including bolstering an alternative identity, minimizing the effects, and transcending (Meisenbach, 2010). Transcendence describes a strategy of highlighting and reframing how a stigma attribute can lead to a higher purpose and a positive result (Meisenbach, 2010). In depression this strategy has been termed resilience, and it has been found to be ineffective, even harmful, to help-seeking intentions (Boardman et al., 2011). The problematic nature of this strategy choice is not only that it is common among college students, as they are in an environment which encourages the pursuit of autonomy and independence, but also that it is derived directly from depression stigma itself (Boardman et al., 2011; Rickwood, 2007; Han et al., 2006).

Other SMC centered research not exclusive to depression stigma also has identified ineffective and effective methods, however since stigma vary dramatically according to type of stigma and the context in which it is expressed, these results must be only very tentatively applied to depressive stigma. This related research suggests that blaming the stigma for unpleasant outcomes has been found to protect the stigmatized individual's self-esteem while bonding with other stigmatized individuals is thought to improve mentoring and peer support (Meisenbach, 2010). Blaming an employer because of the lack of depression awareness could be an example of blaming the stigma, while bonding could involve attending group counseling sessions. Passive acceptance, however, is discouraged (Meisenbach, 2010). Applied to depression, passive acceptance could describe a stigmatized individual not saying anything in a discussion where it is stated that people who are depressed just need to be more positive.

\section{Suggestions for Future Research}

SMC, especially when applied to depression stigma, is still in its infancy and much research is needed to know more about coping strategies. In a general sense, little is known about how the mechanisms in each process works, if different stigma types generate specific strategies, how successful or unsuccessful each strategy is, and how the strategies operate when combined (Meisenbach, 2010). Relevant questions for future research will inquire as to the effectiveness of each response, if the effectiveness is context specific, such as interacting with health professionals versus peers, etc., and if it is identifying what contexts (Barney et al., 2011).

A line of qualitative research, such as narrative inquiry, is needed to begin describing crucial elements of the stigmatized 
experience and how interactions and identities are shaped by SMC (Meisenbach, 2010). Narratives, or stories, are a communicative tool with means for cognitively organizing and interpreting culturally grounded knowledge, experiences, and events (Larkey \& Hecht, 2010; Hecht \& Miller-Day, 2009). It is important to base research on actual experiences, such as those expressed in narratives, in order to more completely understand what coping strategies are used, for what reasons, and with what effects. Narrative inquiry allows for a starting point to base future research directions on that is grounded on the actual experiences of stigmatized individuals. Effectiveness of the strategies can be examined through factors such as subsequent self-esteem and health outcomes (Meisenbach, 2010). In any case, the SMC model and existing research sets a baseline for intervention integration and therefore recommendations for effective implementation are addressed in the next section.

\section{Suggestions for Program Implementation}

Implementing SMC strategies into anti stigma and helpseeking focused programs for depression in college students is a promising tool for utilizing the barriers that stigma poses to help-seeking intentions and behaviors (Boardman et al., 2011). Simply integrating the strategies, however, is not enough. Several aspects are to be considered for successful SMC strategy dissemination including the transportation, audience, demographic, and platform of the implementation. The first is the issue of transportation, and the most effective way to communicate strategy information. Informational approaches on their own have not been proven to be as effective in stigma reduction. As a result, we turn to other methods, such as narrative strategies, which are perceived to be more realistic and easier to relate to (Chang, 2008; Feely et al., 2006).

\section{Transportation Considerations}

Communicating about health requires strategy analysis of effective modes for transferring information. Narrative use is efficacious particularly for health messages involving stigma. Narratives are memorable, efficient, chronologically ordered messages that humans learn at a young age to use to communicate a relationship between events and integrate with previous conceptions in order to form new understandings (Chang, 2008; Kopfman et al., 1998). Narrative health promotion messages allow the experiences of those affected to be expressed in a powerful story format (Hopfer \& Clippard, 2011; Larkey \& Hecht, 2010) that are more effective than other message forms for engaging resistant audiences (Hopfer \& Clippard) such as those involved with stigmas.

Several health initiatives utilize narrative theory and are prime examples for future programs to be modeled after. The practice of narrative competence, otherwise referred to as narrative medicine, among physicians has allowed for increased effectiveness and understanding of medical practice and treatment (Charon, 2001). Motivational interventions, a type of counseling that changes behavior with empathy and reflective listening, have been shown to be successful in decreasing binge drinking among college students and were rated as very favorable among the participants (Borsari \& Carey, 2000). Applied to depression stigma, similar methods may be implemented in a variety of ways, for example, to improve stigma literacy in medical settings or improve coping mechanisms among at-risk students.

Narrative engagement theory suggests that it is important to build curricula from narratives, instead of adapting narratives into new settings (Larkey \& Hecht, 2010; Miller-Day \& Hecht, in press). Eliciting narratives is beneficial both for practical and scholarly aim, but only when done correctly. The main principle of narrative elicitation is that the interview processes should focus on encouraging the interviewee to tell a story, not engage in a discourse (Larkey \& Hecht, 2010). Hopfer and Clippard's (2011) study, involved with the cultural and social framework employed by college aged women in HPV vaccination decisions, followed this principle well and used open ended base questions to encourage story telling (Hopfer \& Clippard, 2011). Another study exploring help-seeking intentions of African American male adolescents employed a similar methodology of interviewing participants who were in treatment and not in treatment about their decisions to seek help in order to gain insight into what factors influences their decisions (Lindsey et al., 2006). Finally, narratives were collected about the social processes of drug offers and became the centerpiece for keepin' it REAL, the most widely disseminated school-based substance abuse curriculum in the world (Miller-Day \& Hecht, in press; Colby et al., in press). All of these provide models for collecting narratives in order to design narrative interventions.

\section{Recipient Considerations: Depressive Status}

Using the SMC framework and related research to develop interventions first requires an acknowledgement of the recipients of the program. Stigma reduction programs typically choose to focus either on nonstigmatized or stigmatized individuals but rarely both. This author argues against an either-or, stigmatized-nonstigmatized mentality and in its place an inclusive perspective that views everyone as potentially stigmatized.

This is a powerful intervention strategy because depressive symptoms exist on a spectrum from mild to severe and stigma is constructed along a two-category system of difference and normalcy. This is complicated by the stigma associated with obtaining treatment for depression, which may be at least partly independent of the stigma attached to the disease. In other words, one might envision a situation in which depression, itself, is not highly stigmatized (i.e., many people are sad) but seeking treatment is stigmatized as a sign of weakness. This is because one key function of stigma messages is to distinguish between group members and nonmembers through the use of group labels and marks (Smith, 2007b). Labels and marks can be as explicit as "crazy" or "emo", but they can also be institutionalized, such as correctly labeling the symptoms of depression or even those who seek treatment (Evans-Lacko et al., 2011). Of course, the opposite situation (stigmatized disease, not treatment) is also possible and presents different challenges. No matter the form, however, the use of marks and labels increase stigmatizing attitudes (Evans-Lacko et al., 2011). As a result, programs that target the "general population" or "nondepressed" individuals in an effort to educate them about depressive illnesses may only reinforce the very stigma construction they are trying to fight. In addition, targeting certain populations based on depressive status often has no impact on actual help-seeking attitudes (Schomerus et al., 2009a).

Combatting stigma and avoiding the use of marks requires elimination of the marks in order to promote inclusion for everyone regardless of depressive status. One strategy for accom- 
plishing this is promoting face-to-face and mediated social contact among stigmatized and non-stigmatized individuals. This strategy has been shown to significantly decrease stigmatized attitudes in other domains (Schulze et al., 2003; EvansLacko et al., 2011; Klin \& Lemish, 2008; Chang, 2008). This strategy requires that individual contact not be written off as unique (i.e., the attribution of the person as an atypical member or even an exception who does not completely belong to the stigmatized group), as often occurs with contact and racial stereotyping, but at the same time must refute stigmatized aspects of depression (Hecht, 1998). An inclusive approach not only relieves the burden (or even stigma) of being the target of prosocial messages but, at the same time including stigmatizing people and stigma targets reduces social distance and increases the likelihood that at-risk, asymptomatic people will seek help should they develop symptoms (Schomerus et al., 2009a).

\section{Demographic Considerations: Race and Gender}

Despite the fact that the rate of mental illness is the same for Americans regardless of race non-white individuals have significantly lower help seeking and detection rates than their white non-Hispanic counterparts (Barney et al., 2011; Schomerus et al., 2009b; Rickwood, 2007; Klein et al., 2011; Kranke et al., 2012; Lindsey et al., 2006). Stigma is a substantial cause of these differences because stigma differs depending on the culture, from health promotion behavior being regarded as a "white" behavior to attitudes that medication is for "crazy people," all of which are informed and reinforced by peers, family, culture, the media, and even the medical community (Kranke et al., 2012; Lindsey et al., 2006; Oyserman et al., 2007). Racial exclusion is even evident in the DSM-IV, which does not differentiate between symptoms specific to various cultures other than that of the dominant white culture, resulting in mis- and under diagnosis among nonwhite patients (Lindsey et al., 2007; Klein et al., 2011; Warren et al., 2010). This tendency is not limited to Black Americans, as Asian Americans also have higher stigmatized perceptions than white Americans with only 3.5\% Chinese Americans willing to seek help and Japanese American students more likely to equate their symptoms with "weak-mindedness" (Han et al., 2006; Chang, 2008). Despite the fact that race is a significant factor in help-seeking rates, culturally grounded psychoeducation models for African Americans are very rare (Rickwood, 2007; Kranke et al., 2012).

Gender must also be considered. Depression has historically been a stigmatized as a biologically female illness and this stigma as persisted to present times in media analysis, diagnosis rates, and manifestation of symptoms (Johansson et al., 2009; Klin \& Lemish, 2008). In addition to perceptions of differences between men and women regarding depression, actual manifestation of symptoms are different with men exhibiting an increase of risk behaviors such as drug and alcohol abuse, and developing antisocial and narcissistic personality traits compared to women who report emptiness and guilt (NIMH, 2011; Michael et al., 2006). While the causes, origins, and validity of these differences are unknown, it remains that the DSM-IV does not classify differing depressive symptoms according to gender thus revealing a gap in diagnostic criteria, public understanding of the criteria, or both.

The effects of the gap includes differences in symptom recognition (men are less likely to recognize depressive symptoms), help seeking rates (men have lower rates), diagnosis (men are less likely to be diagnosed), self-stigmatizing views regarding personal responsibility (men have higher levels), and suicide completion rates (higher for men; Barney et al., 2011; Schomerus et al., 2009b; Rickwood, 2007; Lawlor et al., 2008; Klein et al., 2011; Michael et al., 2006; Elwy et al., 2011; Richards, 2011). These differences are especially relevant to the college student demographic, where in many samples gender differences disappear and even reverse with men in this age group from all ethnic groups more frequently reporting depressive symptoms, but still reluctant to seek help (Eisenberg et al., 2007; Klein et al., 2011; Cassano\& Fava, 2002; Richards, 2011).

Fortunately, while messages are never culture neutral, acknowledging difference by including culturally grounded narratives is effective for culturally diverse groups with various identities that span across gender, age, ethnicity, etc. (Larkey \& Hecht, 2010; Hecht et al., 2003; Oyserman et al., 2007). When the identities embodied the narratives are important to the individual, the health messages endorse behaviors that are not only beneficial to the individual, but also define (or redefine) that individual's identity through social group membership (Oyserman et al., 2007). Depression programs require culturally grounded efforts because depression has historically, socially, and medically been stigmatized as a white woman's illness. Therefore it is important to give voices to those who do not fit into that archetype yet still have depression and narrative health messages provide a promising strategy for accomplishing this goal.

\section{Platform Consideration}

In addition to narrative strategies, recent studies have shown that an Internet platform, accessible by smart phones, is an effective medium to distributing program content, particularly in the case of depression stigma. Among the benefits of an online medium include relevance to the college student demographic, personalization, interaction, anonymity, social proliferation, inexpensively, and efficiency (Morgan et al., 2011; Oh et al., 2009; Lawlor et al., 2008; Rickwood, 2007; Larkey \& Hecht, 2010). Online dissemination has been shown to be effective in improving a variety of health behaviors (Morgan et al., 2011). In 2011 there were over 211 million internet users and the most prevalent users of the Internet are people 25 years old and younger who will first search the internet for health questions (Nielsen, 2011; Oh et al., 2009; Rickwood, 2007). In addition, the internet has been shown to be the ideal medium to reach individuals of various genders, races, and socioeconomic status (Warren et al., 2010). Men favor in person services less favorably than online services and African American students have been found to use the internet for health information more than whites as long as the information is culturally relevant (Oh et al., 2009; Warren et al., 2010).

Online services have also been found to be at least as effective as in person services for symptom reduction, treatment, and counseling (Klin \& Lemish, 2008; Christensen et al., 2004). In an evidence-based review of online mental health programs, six out of the eight online interventions specific to depression were effective at reducing symptoms (Griffiths et al., 2010). One of these programs is the beyond blue campaign, an Australian funded non-profit organization dedicated to the improvement of public perceptions of depression through awareness, support, and destigmatization (Jorm et al., 2006). The program has been 
found to result in higher mental health literacy, more helpseeking intentions, and higher awareness of discrimination $(\mathrm{Oh}$ et al., 2009; Jorm et al., 2006). The prospect of adapting or modeling Depression SMC interventions after campaigns such as beyond blue is promising for depression stigma reduction and increased help seeking behaviors.

\section{Conclusion}

In conclusion, communication perspectives offer promising directions in understanding and influencing health decisions, especially those impacted by stigma such as depression and help seeking in college. While some studies show that strategies such as evading responsibility through provocation and defeasibility can be effective, or reducing disgust through transcendence as ineffective, much of how SMC strategies implicate depression stigma and help seeking rates have not been studied. Narrative inquiry is a promising strategy to begin this much needed research. Prevention programs are strongly urged to incorporate context- and culture-specific SMC because teaching effective personal strategies unrestrained by the depressed/nondepressed binary may increase help-seeking behaviors. Online distribution of the curriculum through narratives allows for fluidity and flexibility in addressing context and culture of the recipients in a way that mimics how stigma function. Utilizing and expanding on all of these findings is promising for the future of stigma reduction and increasing help seeking behavior among depressed individual's in the college student demographic.

\section{REFERENCES}

Barney, L. J., Griffiths, K. M., \& Banfield, M. A. (2011). Explicit and implicit information needs of people with depression: A qualitative investigation of problems reported on an online depression support forum. BMC Psychiatry, 11, 1-11. doi:10.1186/1471-244X-11-88

Barney, L. J., Griffiths, K. M., Jorm, A. F., \& Christensen, H. (2006). Stigma about depression and its impact on help-seeking intentions. Australian and New Zealand Journal of Psychiatry, 40, 51-54. doi:10.1080/j.1440-1614.2006.01741.x

Boardman, F., Griffiths, F., Kokanovic, R., Potiriadis, M., Dowrick, C., \& Gunn, J. (2011). Resilience as a response to the stigma of depression: A mixed methods analysis. Journal of Affective Disorders, 135, 267-276. doi:10.1016/j.jad.2011.08.007

Borsari, B., \& Carey, K. B. (2000). Effects of a brief motivational intervention with college student drinkers. Journal of Consulting and Clinical Psychology, 68, 728-733. doi:10.1037/0022-006X.68.4.728

Cassano, P., \& Fava, M. (2002). Depression and public health: An overview. Journal of Psychosomatic Research, 53, 849-857. doi:10.1016/S0022-3999(02)00304-5

Center for Disease Control (2011). Web-based Injury Statistics Query and Reporting System: Fatal Injury Data. URL (last checked 25 February 2012). http://www.cdc.gov/injury/wisqars/fatal.html

Chang, C. (2008). Increasing mental health literacy via narrative advertising. Journal of Health Communication, 13, 37-55. doi:10.1080/10810730701807027

Charon, R. (2001). Narrative medicine: A model for empathy, reflection, profession, and trust. JAMA, 286, 1897-1902. doi:10.1001/jama.286.15.1897

Christensen, H., Griffiths, K. M., \& Jorm, A. F. (2004). Delivering interventions for depression by using the internet: Randomised controlled trial. British Medical Journal, 328, 265-268. doi:10.1136/bmj.37945.566632.EE

Colby, M., Hecht, M. L., Miller-Day, M., Krieger, J. R., Syverstsen, A. K., Graham, J. W., \& Pettigrew, J. (in press). Adapting school-based substance use prevention curriculum through cultural grounding: An exemplar of adaptation processes for rural schools. American Journal of Community Psychology.

Eisenberg, D., Gollust, S. E., Golberstein, E., \& Hefner, J. L. (2007). Prevalence and correlates of depression, anxiety, and suicidality among university students. American Journal of Orthopsychiatry, 77, 534-542. doi:10.1037/0002-9432.77.4.534

Elwy, A. R., Yeh, J., Worcester, J., \& Eisen, S. V. (2011). An illness perception model of primary care patients' help seeking for depression. Qualitative Health Research, 21, 1495-1507. doi: $10.1177 / 1049732311413781$

Evans-Lacko, S., Brohan, E., Mojtabai, R., \& Thornicroft, G. (2011). Association between public views of mental illness and self-stigma among individuals with mental illness in 14 European countries. Psychological Medicine, 1-12.

Forbes, E. E., \& Dahl, R. E. (2012). Research Review: Altered reward function in adolescent depression: What, when and how? Journal of Child Psychology and Psychiatry, 53, 3-15. doi:10.1111/j.1469-7610.2011.02477.x

Gladstone, T. G., Beardslee, W. R., \& O'Connor, E. E. (2011). The prevention of adolescent depression. Psychiatric Clinics of North America, 34, 35-52. doi:10.1016/j.psc.2010.11.015

Goffman, E. (1963). Stigma: Notes on the management of spoiled identity. Englewood Cliffs, NJ: Prentice-Hall, Inc.

Griffiths, K. M., Farrer, L., \& Christensen, H. (2010). The efficacy of internet interventions for depression and anxiety disorders: A review of randomised controlled trials. Medical Journal of Australia, 192, S11-S4.

Han, D., Chen, S., Hwang, K., \& Wei, H. (2006). Effects of psychoeducation for depression on help-seeking willingness: Biological attribution versus destigmatization. Psychiatry and Clinical Neurosciences, 60, 662-668. doi:10.1111/j.1440-1819.2006.01580.x

Hankin, B. L. (2006). Adolescent depression: Description, causes, and interventions. Epilepsy and Behavior, 8, 102-114. doi:10.1016/j.yebeh.2005.10.012

Hecht, M. L. (Ed.) (1998). Communicating prejudice. Newbury Park, CA: Sage.

Hecht, M. L., Marsiglia, F. F., Elek, E., Wagstaff, D. A., Kulis, S., Dustman, P., \& Miller-Day, M. (2003). Culturally grounded substance use prevention: An evaluation of the keepin' it R.E.A.L. curriculum. Prevention Science, 4, 233-248. doi:10.1023/A:1026016131401

Hecht, M. L., \& Miller-Day, M. (2009). The drug resistance strategies project: Using narrative theory to enhance adolescent's communication competence. In L. Frey, \& K. Cissna (Eds.), Routledge Handbook of Applied Communication (pp. 535-557). New York and London: Routledge.

Hopfer, S., \& Clippard, J. R. (2011). College women's HPV vaccine decision narratives. Qualitative Health Research, 21, 262-277. doi: $10.1177 / 1049732310383868$

Johansson, E. E., Bengs, C., Danielsson, U., Lehti, A., \&Hammarström, A. (2009). Gaps between patients, media, and academic medicine in discourses on gender and depression: A metasythesis. Qualitative Health Research, 19, 633-644. doi:10.1177/1049732309333920

Jorm, A. F., Christensen, H., \& Griffiths, K. M. (2006). Changes in depression awareness and attitudes in Australia: The impact of beyondblue: The national depression initiative. Australian and New Zealand Journal of Psychiatry, 40, 42-46.

Jorm, A. F., \& Griffiths, K. M. (2008). The public's stigmatizing attitudes towards people with mental disorders: How important are biomedical conceptualizations? Acta Psychiatry Scandinavica, 118, 315-321. doi:10.1111/j.1600-0447.2008.01251.x

Klein, M. C., Ciotoli, C., \& Chung, H. (2011). Primary care screening of depression and treatment engagement in a university health center: A retrospective analysis. Journal of American College Health, 59, 289-295. doi:10.1080/07448481.2010.503724

Klin, A., \& Lemish, D. (2008). Mental disorders stigma in the media: Review of studies on production, content, and influences. Journal of Health Communication, 13, 434-449. doi: 10.1080/10810730802198813

Kopfman, J. E., Smith, S. W., Ah Yun, J. K., \& Hodges, A. (1998). Affective and cognitive reactions to narrative versus statistical evi- 


\section{E. REICHERT}

dence organ donation messages. Journal of Applied Communication Research, 26, 279-300. doi:10.1080/00909889809365508

Kranke, D., Guada, J., Kranke, B., \& Floersch, J. (2012). What do African American youth with a mental illness think about helpseeking and psychiatric medication? Origins of stigmatizing attitudes. Social Work in Mental Health, 10, 53-71. doi:10.1080/15332985.2011.618076

Kravitz, R. L., Paterniti, D. A., Epstein, R. M., Rochlen, A. B., Bell, R. A., Cipri, C., \& Fernandez y Garcia, E. (2011). Relational barriers to depression help-seeking in primary care. Patient Education and Counseling, 82, 207-213. doi:10.1016/j.pec.2010.05.007

Larkey, L. K., \& Hecht, M. (2010).A Model of Effects of Narrative as Culture-Centric Health Promotion.Journal of Health Communication, 15, 114-135. doi:10.1080/10810730903528017

Lawlor, E., Breslin, J. G., Renwick, L., Foley, S., Mulkerrin, U., Kinsella, A., \& Turner, N. (2008). Mental health literacy among Internet users. Early Intervention in Psychiatry, 2, 247-255. doi:10.1111/j.1751-7893.2008.00085.x

Lindsey, M. A., Korr, W. S., Broitman, M., Bone, L., Green, A., \& Leaf, P. J. (2006). Help-seeking behaviors and depression among African American adolescent boys. Social Work, 51, 49-58. doi:10.1093/sw/51.1.49

Meisenbach, R. J. (2010, August). Stigma management communication: A theory and agenda for applied research on how individuals manage moments of stigmatized identity. Journal of Applied Communication Research, 38, 268-292. doi:10.1080/00909882.2010.490841

Michael, K. D., Huelsman, R. J., Gerard, C., Gilligan, T. M., \& Gustafson, M. R. (2006). Depression among college students: Trends in prevalence and treatment seeking. Counseling and Clinical Psychology Journal, 3, 60-70.

Miller-Day, M., \& Hecht, M. L. (in press). Narrative means to preventative ends: A narrative engagement approach to adolescent substance use prevention. Health Communication.

Morgan, A. J., Jorm, A. F., \& Mackinnon, A. J. (2011). Protocol for a randomised controlled trial investigating self-help email messages for sub-threshold depression: The Mood Memos study. Trials Journal, 12, 1-9.

Moyer-Gusé, E., \& Nabi, R. L. (2010). Explaining the effects of narrative in an entertainment television program: Overcoming resistance to persuasion. Human Communication Research, 26, 26-52. doi:10.1111/j.1468-2958.2009.01367.x

National Institute of Mental Health (n.d.) Depression and College Students. National Institues of Health. URL (last checked 25 February 2012).

http://www.nimh.nih.gov/health/publications/depression-and-college -students/index.shtml

National Institute of Mental Health (2011). Depression. National Institutes of Health. URL (last checked 25 February 2012).

http://www.nimh.nih.gov/health/publications/depression/index.shtml

Nielsen (2011). Consumer Usage Report. http://www.nielsen.com/content/dam/corporate/us/en/reports-downlo ads/2011-Reports/StateofMediaConsumerUsageReport.pdf

Oh, E., Jorm, A. F., \& Wright, A. (2009). Perceived helpfulness of websites for mental health information. Social Psychiatry and Psychiatry Epidemiology, 44, 293-299. doi:10.1007/s00127-008-0443-9

Oyserman, D., Yoder, N., \& Fryberg, S. A. (2007). Identity-based motivation and health. Journal of Personality and Social Psychology, 93, 1011-1027. doi:10.1037/0022-3514.93.6.1011

Richards, D. (2011). Prevalence and clinical course of depression: A review. Clinical Psychology Review, 31, 1117-1125. doi:10.1016/j.cpr.2011.07.004

Rickwood, D. J., Deane, F. P., \& Wilson, C. J. (2007). When and how do young people seek professional help for mental health problems? Medical Journal of Australia, 187, S35-S39.

Schomerus, G., Matschinger, H., \& Angermeyer, M. C. (2009a). Attitudes that determine willingness to seek psychiatric help for depression: A representative population survey applying the Theory of Planned Behavior. Psychological Medicine, 39, 1855-1865. doi:10.1017/S0033291709005832

Schomerus, G., Matschinger, H., \& Angermeyer, M. C. (2009b). The stigma of psychiatric treatment and help-seeking intentions for depression. European Archives of Psychiatry and Clinical Neuroscience, 259, 298-306. doi:10.1007/s00406-009-0870-y

Schulze, B., Richter-Werling, M., Matschinger, H., \& Angermeyer, M. C. (2003). Crazy? So what! Effects of a school project on students' attitudes towards people with schizophrenia. Acta Psychiatrica Scandinavica, 107, 142-150. doi:10.1034/j.1600-0447.2003.02444.x

Smith, R. A. (2007a). Language of the lost: An explication of stigma communication. Communication Theory, 17, 462-485. doi:10.1111/j.1468-2885.2007.00307.x

Smith, R. (2007b). Media depictions of health topics: Challenge and stigma formats. Journal of Health Communication, 12, 223-249. doi:10.1080/10810730701266273

Ting, L. (2011). Depressive symptoms in a sample of social work students and reasons preventing students from using mental health services: An exploratory study. Journal of Social Work Education, 47, 253-268. doi:10.5175/JSWE.2011.200900092

Warren, J. R., Kvasny, L., Hecht, M. L., Burgess, D., Ahluwalia, J. S., \& Okuyemi, K. S. (2010). Barriers, control and identity in health information seeking among African American women. Journal of Health Disparities Research and Practice, 3, 68-90.

Yap, M., Wright, A., \& Jorm, A. F. (2011). The influence of stigma on young people's help-seeking intentions and beliefs about the helpfulness of various sources of help. Social Psychiatry and Psychiatry Epidemiology, 46, 1257-1265. doi:10.1007/s00127-010-0300-5

Zivin, K., Eisenberg, D., Gollust, S. E., \& Golberstein, E. (2009). Persistence of mental health problems and needs in a college student population. Journal of Affective Disorders, 117, 180-185. doi:10.1016/j.jad.2009.01.001 